\title{
27. Experimental Studies on the Treatment of Carotid Arterial Thrombosis in Dogs
}

\author{
Hajime Handa, Kouzo Yoshida, Jyoji Handa, Takuro Takase \\ and Akinori Kondo \\ First Surgical Division, Kyoto University Medical School,
}

Although reconstructive operations have been widely recognized as the bes treatment for carotid arterial occlusions, circulation is successfully restored in onl: $30 \%$ of cases with the complete occlusions. In the present study, therefore, the treatment with thrombolytic agents and/or anticoagulants have been compared witl that of thrombectomy.

From our experimental studies with 5 different methods of producing carotic arterial thrombosis in dogs, it was found that thrombotic occlusion was producer by the method with electric current in $92.5 \%$ of cases. Moreover, the thrombotic occlusion produced by this method is likely to become complete in course of days.

Using above-mentioned carotid arterial thrombosis, thrombectomy, anti coagulants and thrombolytic agents have been tried immediately, the 2nd, the 3 rd and the 5 th day respectively.

The following results have, so far, been obtained.

1) The restoration of blood circulation was obtained by thrombolytic agent: without producing any noticeable side effects including cerebral hemorrhage ir almost the same ratio (40-62.5\%) as by thrombectomy in cases within two days

2) The restoration of blood circulation was obtained only in $16.7 \%$ eithes by thrombectomy or thrombolytic agents in cases after 3 days.

3) Such a poor result was restored to $80 \%$ by thrombectomy followed b: heparin administration for 48 hours in cases of 3 days.

\section{The Time-Development of the Experimental Cerebro Vascular Disease}

Toyozo Aizawa, Yuichiro Goto, Tsuneo Hasegawa, Goro Araki, Hisashi Takitsuka, Akinori Kanda, Koyu Mori, Keiichi Murakami,

Nobuhisa Sato, Keiji Yoshida, Shiro Nagano, Sadao Hori, Masahiro KATo and Katsuya KoJima

Keio University School of Medicine

Cerebral embolism was produced by the injection of Gelatin-Indian Ink clot or coagulated blood clots via the internal carotid artery on dogs, and then the dog 
were sacrificed half hour, one hour, two hours, four hours, eighteen hours and twenty four hours after the operation. Some of them were alived for 2 days to 20 days after the operation. We examined these groups of cerebral lesions and cerebral blood vessels with the histological method. Thirty minutes after the operation, the cerebral vascular lesions and cerebral lesions were not found by the ordinal histological methods.

One to two hours after the operation, the section revealed partially hemorrhage via vascular wall into the perivascular spaces but there were no vascular changes.

Four hours after the injection, the section showed the perivascular transudation of blood cells, the exsudation of serum and indian ink, however the angionecrosis could not be found.

Eighteen hours after the injection the section revealed the remarkable appearance of fibrin in perivascular spaces, there were also hemorrhage into the perivascular spaces, extending to the local cerebral tissue. Angionecrosis were found in some of the sections.

Twenty four hours after the operation the perivascular hemorrhage and white infarction were frequently found. There were proliferation of glia cells at the peripheral areas of the cerebral lesions and angionecrosis was common in the hemorrhagic infarction.

Two days after the operation, the section showed the new formation of blood vessels, the proliferation of glia cells and the appearance of fat granulated cells.

According to the changes of the above described, at the first the increased permeability of blood vessel could be occurred, the second hemorrhage could be followed, and then fibrin could be appear in cerebral vascular wall producing angionecrosis.

\title{
29. Anterior Cerebral Artery, Thrombosis and Hypoplasia of its Pars Circularis
}

\author{
Soji SuzuKI \\ Dept. of Radiology, Tokyo Medical \& Dental University \\ School of Medicine
}

It is well-known that the circle of Willis has various anomalies in its anatomical configuration and the so-called "textbook" circle of Willis is encountered in only approximately fifty per cent of the normal population. The string-like Pars Circularis of anterior cerebral artery is rather uncommon among the anomalies but should be always born in mind, because the overlook of the anomaly may lead 